\title{
Was ist Liebe? Ein wirklichkeitssoziologischer Zugang zu einem vernachlässigten Thema ${ }^{1}$
}

\section{Max Haller}

Zusammenfassung: „Liebe“ ist ein in der Soziologie stark unterbelichtetes Thema. Ausgehend von einer Dokumentation dieses Faktums in vier großen soziologischen Handbüchern wird in diesem Beitrag argumentiert, dass man Liebe unter einer Weberschen Perspektive als emotionales Handeln betrachten kann. Unter besonderem Bezug auf die erotisch-sexuell fundierte Partnerbeziehung werden vier Aspekte für eine soziologische Analyse von Liebe als wesentlich erachtet: die emotionale Komponente, der kognitive Aspekt, die Handlungskomponente (wichtig dabei: der Raum- und Zeitbezug) und der ethisch-normative Aspekt. Im Anschluss daran wird kurz diskutiert, welche Bedeutung dem Konzept der ,romantischen Liebe“ heute noch zukommt, und wie die Zukunft von Liebesbeziehungen aussieht.

Schlüsselwörter: Liebe, soziale Emotionen, Soziologie als Wirklichkeitswissenschaft, Max Weber

\section{What is love? A Weberian approach to a negelced sociological topic}

\begin{abstract}
Love“ is a topic largely neglected in sociology. This article starts from a documentation of this fact in four large sociological handbooks and develops the argument that love can be analysed from a Weberian theoretical perspective as emotional social action. Focussing upon the erotic-sexual love relationship. It is argued that four aspects are crucial in this perspective: Love as emotion, as social action (in-
\end{abstract}

1 Wichtige Anregungen für diesen Aufsatz erhielt ich von Susan Mlangwa (Dar es salaam, Tanzania) und Bernadette Müller-Kmet (Innsbruck).

M. Haller $\bowtie$

Universität Graz, Institut für Soziologie

Universitätsstraße 15/G4, 8010 Graz, Österreich

E-Mail: max.haller@uni-graz.at 
cluding the relation to space and time), as a cognition and as an ethical-normative issue. Then the question of present-day relevance of the romantic love ideal is raised. In the conclusion, some future prospects for love in partnership relationships are discussed.

Keywords: love, social emotions, theory of social reality, Max Weber

\section{Zur Relevanz der Fragestellung}

Sieht man sich Literatur und andere geistige Erzeugnisse zum Thema „Liebe“ an, so fällt eine starke Kluft auf: Das Thema „Liebe“ spielt in Romanen und Lyrik, in Theater und Film und in der bildenden Kunst eine überragende Rolle ${ }^{2}$, und es gibt auch eine umfangreiche (tiefen-) psychologische und populäre Ratgeberliteratur zum Thema. Untersucht man Texte aller Art, so ist „Liebe“ einer der am häufigsten vorkommenden Begriffe (Kelley 1993). In der Soziologie stellt Liebe jedoch fast nur ein Randthema dar, obwohl diese Disziplin zu ihrer Analyse prädestiniert erscheint (vgl. auch Goode 1974; Hahn und Burkart 2000). Dies gilt selbst für jene soziologischen Teildisziplinen, in denen sie eigentlich eine zentrale Rolle spielen sollte, der Forschung zu Partnerschaft, Ehe und Familie. Tabelle 1 zeigt die Häufigkeit der Behandlung des Themas in vier großen Handbüchern zu diesem Bereich; dabei werden auch weitere positiv konnotierte Begriffe - wie bonding, affection, happiness bzw. Fürsorge, Bindung, Zufriedenheit - betrachtet. Diese Begriffe werden der Häufigkeit anderer gegenübergestellt, welche negative Aspekte von Ehe- und Familienbeziehungen indizieren, wie violence, conflict, power, abuse, stress bzw. Gewalt, Konflikt, Missbrauch.

Das Ergebnis ist kaum zu glauben: Der Begriff Liebe taucht nur in einer verschwindend geringen Häufigkeit auf im Vergleich zu jenen Begriffen, die problembeladende, konflikthafte und ausbeuterische Beziehungen indizieren; im neueren englischsprachigen Handbuch kommt er überhaupt nicht mehr vor. Auch Begriffe wie enge Beziehung (bonding), Fürsorge und $\mathrm{Zu}-$ neigung, Zufriedenheit und Glück sind vergleichsweise selten. Mit Abstand am häufigsten sind dagegen Begriffe wie Macht und Gewalt, Konflikt und Stress, Missbrauch, Aggression und Unterdrückung. Man könnte meinen, Ehe und Familie heute seien gesellschaftliche Kontrollund Zwangseinrichtungen, geprägt von Kampf und Gewalt gegen Frauen, Vernachlässigung und Misshandlung von Kindern und nicht jene Institutionen, welche dem Menschen den wichtigsten sozialen Rückhalt bieten, ihn vor Vereinsamung und Selbstmord schützen, wie Emile Durkheim schon 1897 nachgewiesen hat und seither zahllose Studien bestätigt haben (vgl. z.B.

2 Thomas Anz, „Liebe, Ehe und Familie in der Literatur“, in: http://www.literaturkritik.de/public/rezension.php?rez_ $\mathrm{id}=5626(29.4 .2016)$. 
Tabelle 1: Die am häufigsten vorkommenden negativen und positiven Begriffe in Bezug auf Partner- und Familienbeziehungen in deutsch- und englischsprachigen Handbüchern zu Ehe und Familie

\begin{tabular}{|c|c|c|c|c|}
\hline \multirow{4}{*}{$\begin{array}{l}\text { Sussman/Steinmetz, } \\
\text { Handbook of Marriage } \\
\text { and the Family (1987) }\end{array}$} & \multicolumn{2}{|l|}{ Negativ konnotierte Begriffe } & \multicolumn{2}{|l|}{ Positiv konnotierte Begriffe } \\
\hline & Power (parental, marital) & 390 & bonding & 50 \\
\hline & conflict & 130 & affection & 40 \\
\hline & abuse (spouse, child) & 106 & rewards & 40 \\
\hline \multirow{8}{*}{$\begin{array}{l}\text { Peter/Bush, Handbook } \\
\text { of Marriage and the } \\
\text { Family (2013) }\end{array}$} & stress & 105 & love & 14 \\
\hline & violence & 90 & happiness & 14 \\
\hline & & & altruism & 3 \\
\hline & $\begin{array}{l}\text { violence, coercion, } \\
\text { aggression }\end{array}$ & 76 & $\begin{array}{l}\text { (economic) well-being, } \\
\text { happiness, satisfaction }\end{array}$ & 3 \\
\hline & $\begin{array}{l}\text { (child) abuse, maltreatment, } \\
\text { neglect }\end{array}$ & 46 & parental support & 13 \\
\hline & stress & 36 & strength, resilience & 1 \\
\hline & oppression & 18 & $\begin{array}{l}\text { (couple, parent) simpatia, } \\
\text { respeto }\end{array}$ & 4 \\
\hline & conflict & 15 & love & 0 \\
\hline \multirow{4}{*}{$\begin{array}{l}\text { Nave-Herz/Markefka, } \\
\text { Handbuch d. Familien- } \\
\text { u. Jugendforschung } \\
\text { (1989) }\end{array}$} & Macht & 23 & Liebe & 8 \\
\hline & Gewalt & 23 & Fürsorge & 4 \\
\hline & Verhaltensauffälligkeiten & 20 & Zufriedenheit & 6 \\
\hline & & & Bindung & 2 \\
\hline \multirow{3}{*}{$\begin{array}{l}\text { Hill/Kopp, Handbuch } \\
\text { Familiensoziologie } \\
(2015)\end{array}$} & Konflikt & 23 & Liebe & 5 \\
\hline & Gewalt & 12 & Zufriedenheit, Glück & 0 \\
\hline & Macht & 8 & & \\
\hline
\end{tabular}

Quelle: Einträge in den Stichwortverzeichnissen der vier Handbücher (als Einzelbegriffe oder in Zusammensetzung mit anderen Begriffen)

Brockmann und Klein 2004; Dette-Hagenmeyer und Reichle 2015). Ist es nur Selbsttäuschung, wenn Befragte über alle Schichten hinweg die Bereiche Partnerschaft und Familie/Kinder als die wichtigsten in ihrem Leben bezeichnen, und $\mathrm{zu}$ fast $90 \%$ mit diesen sozialen Beziehungen zufrieden oder sehr zufrieden sind, wie Bevölkerungsumfragen zeigen (Schulz 1983; Haller 2005; Schulz und Pichler 2005)? 
Es gibt eine umfangreiche weitere Literatur, die sich mit dem Thema Liebe im weiteren Sinne beschäftigt, aber auch hier werden eher problematische Aspekte thematisiert oder solche, die nur peripher mit dem Thema Liebe zu tun haben. Eine feministische Autorin wie Shulamith Firestone (1975) sieht hier den Schlüssel zur Unterdrückung der Frauen; die männliche Herrschaft und Kultur ist auf der Liebe der Frauen und deren Hingebung für die Männer aufgebaut (vgl. dazu auch Illousz 2011, S. 15ff.). Auch weniger radikalen feministischen Arbeiten zur Geschlechtersoziologie wird auf Liebe nur nebenbei und wenn, dann eher kritisch Bezug genommen. So wird das Ideal der romantischen Liebe als etwas Oktroyiertes bezeichnet: „As the ideal of romantic love is spread around the world by religion, advertising and other cultural pressures, it comes into conflict with other ways of forming new households [...]" (Connell and Pearse 2015, S. 82). Umfangreiche soziologische Literatur gibt es zum Thema der schichtspezifischen Partnerwahl, den „Heiratsmarkt“ und die Muster von Homogamie und Heterogamie nach sozialstatistischen Merkmalen der Partner (vgl. z.B. Klein 2001; Haller 2008, S. 319-326). Diese Forschungen sind relevant, wenn es um Rahmenbedingungen geht, innerhalb derer sich Liebesbeziehungen entwickeln können und vor allem im Hinblick auf die Frage der Reproduktion sozialer Schichtung und Ungleichheit über die Generationen (Haller 1983). Das Thema „Liebe“ thematisieren all diese Studien aber nicht - wenn sie nicht gar irreführend folgern, Liebe sei ein „Trugschluss“, da die Partnerwahl ja stark durch die Sozialstruktur bestimmt werde. Allerdings hat das Vordringen eines neuen, umfangreichen Heiratsmarktes (insbesondere auch im Internet), auf welchem Aussehen und sexyness eine große Rolle spielen, dazu geführt, dass Partnersuche und -wahl zunehmend unter Leistungsaspekten gesehen werden und damit individualisiert und kommerzialisiert werden (Illousz 2011, S. 0-111). ${ }^{3}$ Es gibt natürlich auch Soziologinnen und Soziologen, die sich explizit mit dem Thema Liebe ${ }^{4}$ heute befassen (vgl. u.a. Luhmann 1982; Beck/Beck-Gernsheim 1990; Hahn und Burkart 2000; Lenz 3003). Meiner Meinung haben aber auch sie keine Perspektive auf Liebe entwickelt, wie sie sich aus dem Weberschen Ansatz der Soziologie als Wirklichkeitswissenschaft ableiten lässt. ${ }^{5}$ Kaum fündig wird man auch in dem inzwischen etablierten Forschungsbereich Soziologie der Emotionen. In der Einführung von Helena Flam (2002) wird das Thema „Liebe“ nur in Diskussionen von Klassikern behandelt, nicht jedoch im systematischen Teil; hier geht es nur um Gefühle im Zusammenhang mit Arbeit, Geld und Politik.

Wie ist diese Diskrepanz in der Behandlung von Liebe in Kunst, Literatur und populärwissenschaftlichen Schriften im Unterschied zur Soziologie zu erklären? Man kann zumindest vier

3 In mittleren und höheren Schichten hat der Heiratsmarkt aber auch schon in den 1970er Jahren eine erhebliche Rolle gespielt (vgl. Haller 1980).

4 Vgl. Thomas Klein, „Der Trugschluss der Liebe“, Universität Heidelberg, Uni-Spiegel 1/2000 (3.5.2016). Dieser Titel ist sicherlich mehr als ein Aufhänger zu verstehen denn als ernstgemeinte These.

5 Allerdings hat auch Weber selbst zum Thema Partnerliebe sehr wenig geschrieben, nicht zuletzt vielleicht wegen seiner persönlichen massiven Probleme mit Erotik und Sexualität in der Ehe (vgl. dazu Radkau 2005). 
Gründe dafür anführen (vgl. dazu auch Burkart 2000, S. 8, 174-175). Ein erster Grund ist wohl der, dass die Liebe etwas Geheimnisvolles zu sein scheint, etwas, dem sich zwar Literatur und schöne Künste widmen können, das aber mit den Mitteln der rational-abstrakten Wissenschaft nicht erfasst werden kann.

Zum Zweiten muss man zugeben, dass Vernachlässigung, Missbrauch und Gewalt in Familien nichts Seltenes sind, wie schon Max Weber (1964, S. 30) schrieb. Die These, dass sich Frauen in die größte Gefahr begeben, wenn sie zu ihrem Mann nach Hause zurückkehren und die Tür hinter sich abschließen, ist nicht unbegründet; von allen Gewalttaten gegen Frauen erfolgt ein großer Teil zuhause. Zum Dritten ist klar, dass auch die Darstellung der Liebe in Literatur und Kunst sich nicht auf harmonische und glückliche Beziehungen und Ehen konzentriert, sondern gerade die Spannungen und Irrungen, Probleme und Dramen im Zusammenhang mit Liebe, Liebeskummer und -schmerz herausarbeitet. Auch in der Popmusik werden vielfach gescheiterte und unglückliche Liebesbeziehungen dargestellt (Scheff 2011). Positive familiäre Affektivität findet ,[...] in der Gesamtkultur wenig Stütze, Symbolik und Auszeichnung [...]“ (Rosenmayr 1986, S. 73). Viertens ist evident, dass sich in der literarischen Moderne auch die Darstellung von Liebe, Ehe und Familie gewandelt hat: das harmonische Leitbild wird zunehmend bezweifelt, Männer und vor allem Frauen erscheinen ihren Zwängen unterworfen und als Opfer familiärer Desaster. So schreiben auch Ulrich und Elisabeth Beck-Gernsheim (1990, S. 84), sie interessieren sich nicht für ,, [...] die Idealformen und möglichen Wunder [...]“ in Zweierbeziehungen, sondern für „,...] die andere Seite, das vielfache Scheitern von Beziehungen heute $[\ldots]^{\text {“. }}$.

Meine These lautet, dass diese Vernachlässigung des Themas „Liebe“ nicht nur eine große Forschungslücke der Soziologie darstellt, sondern auch bedeutsame praktische Auswirkungen hat insofern, als sie zu einer übertrieben negativ-pessimistischen Sicht von Partner-, Ehe- und Familienbeziehungen führt.

\section{Was ist Liebe? Definitionen der Alltagssprache}

Liebe ist eine universelle, tiefgehende alltägliche wie auch außeralltägliche menschliche Erfahrung; für sie gibt es auch in allen Sprachen eigene Begriffe - oft nicht nur einen, sondern eine ganze Reihe, welche unterschiedliche Formen und Nuancen der Liebe zum Ausdruck bringen. ${ }^{6}$

6 In Swahili, einer verbreiteten Umgangssprache in Ostafrika, gibt es dafür mindestens neun; die Begriffe lauten: pendo, upendo, upenzi, huba (Liebe); mapendezi (Liebe, Sehnsucht); kipendo (Zuneigung); ashiki (Neigung, Trieb, Gier); hebu (Ergebenheit, glücklich sein, Freundschaft); hashiki (heiße Liebe); vgl. Suaheli online Wörterbuch; http://www.suaheli.eu/ (30.4.2016). 
Es scheint daher angebracht, sich kurz anzusehen, wie Liebe in allgemeinen Wörterbüchern und Lexika definiert wird. In wikipedia etwa heißt es: Liebe ist...

„[...] ein starkes Gefühl, mit der Haltung inniger und tiefer Verbundenheit zu einer Person, die den Zweck oder den Nutzen einer zwischenmenschlichen Beziehung übersteigt und sich in der Regel durch eine entgegenkommende tätige Zuwendung zum anderen ausdrückt."

In einem allgemeinen Lexikon wird Liebe wie folgt definiert:

„Liebe, ein Begriff, mit dem eine Vielfalt von Gefühlen der Zuneigung charakterisiert wird, die auf die Vereinigung mit dem geliebten Objekt zielen. Man unterscheidet die personenbezogene Liebe zu einem Partner, die meist die Sexualität mit einschließt, oder zu Eltern, Freunden, Geschwistern und anderen Menschen und die objektbezogene Liebe zur Natur, zur Freiheit, zum Eigentum u.a. ${ }^{\text {" }}$

In einem älteren Sprach-Brockhaus kommen weitere Facetten des Begriffs zur Sprache:

„Liebe, 1) opferbereite Gefühlsbindung, Zuneigung, im engeren Sinne; geschlechtsgebundene Gefühlsbeziehung; die Liebe des Vaters [...] zum Kinde hat; die Liebe zum Vater, für den Vater, die Liebe des Kindes; Liebe zum Vaterland, zur Tugend; freie Liebe; außerehel. Verhältnis. 2) Erbarmen, Mildtätigkeit: Werke der Liebe [...] 3) Freundlichkeit: tun Sie mir die Liebe [...] und kommen Sie mit. 4) Geliebter Mensch: meine erste Liebe; eine alte Liebe von mir." ${ }^{\text {"9 }}$

Wir können die folgenden drei Aspekte als wichtig erkennen: 1) Liebe ist ein Gefühl, das eine positive Beziehung indiziert; 2) Liebe kann sich nicht nur auf Menschen, sondern auch auf Objekte ${ }^{10}$ beziehen; und 3) sie hat einen Handlungsbezug. Im den folgenden Ausführungen lege ich den Schwerpunkt auf die erotisch-sexuelle Partnerbeziehung, gehe ab und zu aber auch auf andere Liebesbeziehungen ein; die These lautet, dass sie alle einen gemeinsamen Kern besitzen.

7 https://de.wikipedia.org/wiki/Liebe (30.4.2016).

8 Bertelsmann Neues Lexikon in 10 Bänden, Bd.6, S. 64; Gütersloh: Bertelsmann Lexikon Verlag.

9 Der Sprach-Brockhaus. 1958. Wiesbaden: F.A. Brockhaus, S. 403.

10 Tilman Habermas (1999) hat in einem umfangreichen Werk dargestellt, dass auch persönliche Objekte (wie Musikinstrumente, Kleidungsstücke, Einrichtungsgegenstände) und Tiere vielfältige Funktionen (wie Selbstdarstellung, Selbstkommunikation, Erinnerung, Autonomiestärkung) für Menschen erfüllen können. Auch aus dieser Sicht erscheint der Fokus auf „Liebe“ anstatt auf „persönliche Beziehungen“ (wie bei Lenz 2003) als umfassender und konkreter, also stärker ,wirklichkeitssoziologisch“. 


\section{Die vier zentralen Komponenten von Liebe}

Ich gehe davon aus, dass vier Komponenten wesentlich sind, um liebesbasiertes soziales Handeln bzw. soziale Beziehungen soziologisch adäquat erfassen zu können (vgl. Übersicht_1): Es sind dies die emotionalen Aspekte, der Aspekt des sozialen Handelns, der kognitive Aspekt und der ethisch-normative Aspekt. Die These lautet, dass diese Aspekte in fast allen Liebesbeziehungen eine wichtige Rolle spielen.

Übersicht 1: Die vier zentralen Aspekte von Liebe aus wirklichkeitssoziologischer Sicht

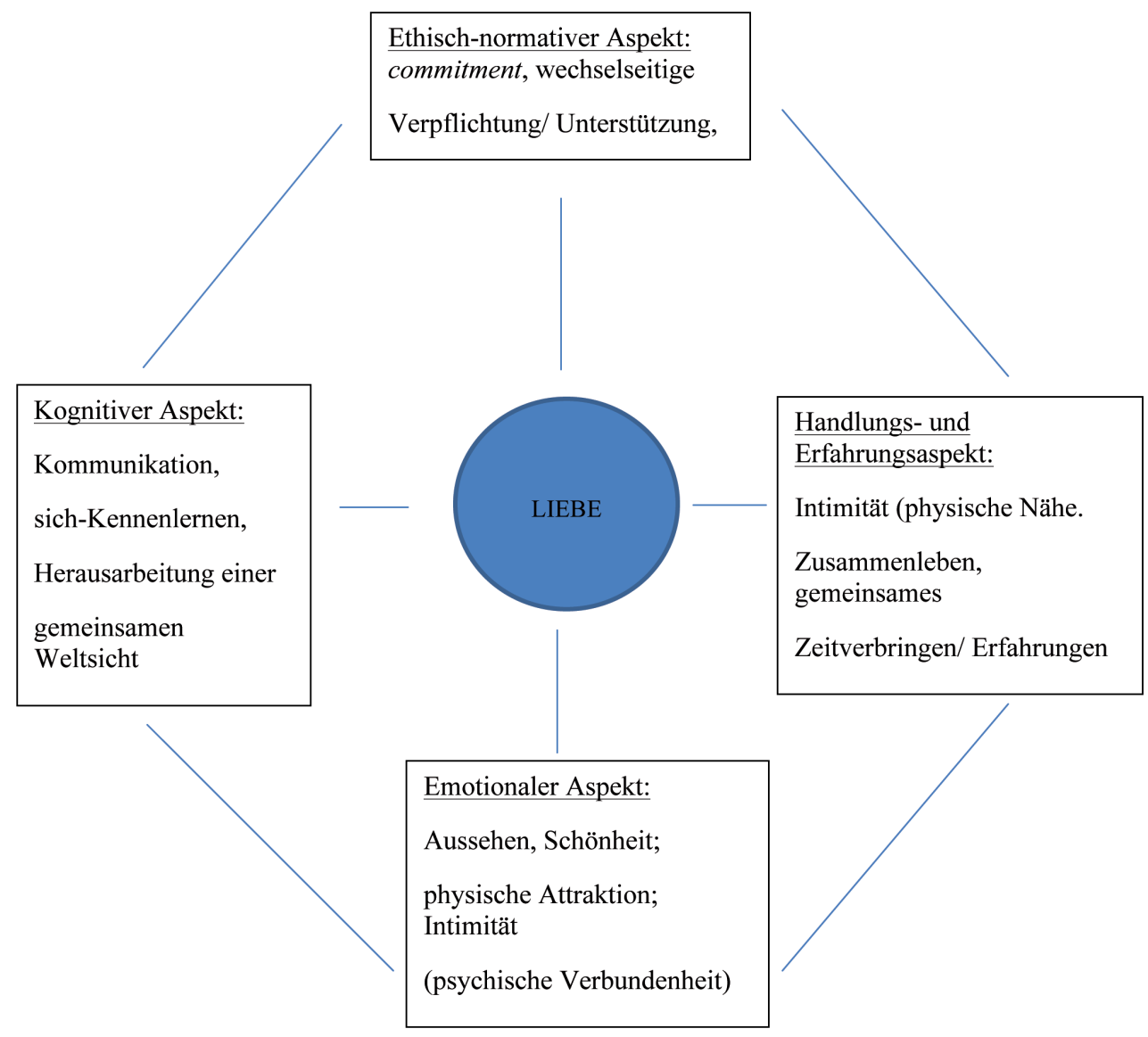


Sie tun dies in verschiedenen Arten von Liebesbeziehungen in unterschiedlicher Weise bzw. Gewichtung. Der emotionale Aspekt ist am wichtigsten in der erotisch-sexuellen Partnerbeziehung; der Aspekt von Handlungen und Erfahrungen spielt in der Mutter-Kind und Vater-Kind Beziehung die stärkste Rolle. Sie ist auch zentral für die Geschwisterbeziehung, die eine sehr wichtige Form von Beziehungen auch in modernen, mobilen Gesellschaften darstellt (Haller 2013). Der kognitive Aspekt ist zentral in Freundschaftsbeziehungen, in denen es um ähnliche Wertorientierungen und Vorlieben geht (Heidbrink 2007) und vielleicht auch am Beginn von Partnerbeziehungen, wenn es auch um eine möglichst vorteilhafte Selbstpräsentation geht. Der ethisch-normative Aspekt ist essentiell in der Nächsten- und Gottesliebe; in dieser Form von Liebe, die im Christentum zentrale Bedeutung hat, aber auch von allen Weltreligionen mehr oder weniger ausdrücklich gefordert wird, ist der Handlungsbezug am offenkundigsten: Man soll nicht nur Nahestehenden helfen bzw. Personen, die einem sympathisch sind, sondern ohne Unterschied allen Menschen, die der Hilfe bedürfen. ${ }^{11}$ Für den buddhistischen Dalai Lama (2016, S. 16) ist die „echte Religion“ nichts anderes als Liebe und Mitgefühl, die Bereitschaft, die Rechte anderer Menschen zu respektieren.

\section{Liebe als Emotion und Gefühl ${ }^{12}$}

Liebe ist zuallererst ein Gefühl, eine Emotion, die auch mit physiologischen Prozessen verbunden ist (Roth 2003): Es impliziert ein sich zum anderen hingezogen fühlen, sie oder ihn mehr oder weniger bedingungslos akzeptieren, im Extremfall eine durch Vernunft nicht mehr fassbare Passion oder Leidenschaft. ${ }^{13}$ Starke Liebe ist auch mit tiefem Glücksgefühl bzw. - im Falle einer Enttäuschung - mit Unglücklichsein und Verzweiflung verbunden. Erwiderte Liebe bedeutet eine enorme Erhöhung des Selbstwert-Gefühls, erzeugt daher Stolz; zurückgewiesene Liebe bedeutet eine Verletzung des Selbstwertgefühls, führt daher zu Scham. ${ }^{14}$ David Hume (1978, S. 60ff.) sah eine Parallelität zwischen Stolz und Scham einerseits, Liebe und Hass andererseits: aus Liebe zu jemandem kann Stolz entstehen und aus Stolz auf jemanden, der tugendhaft ist, Liebe; dagegen erzeugt lasterhaftes Verhalten eines anderen Menschen Hass auf diesen.

Die neurowissenschaftliche Hirnforschung zeigt, dass Liebe ,als genetisch kontrollierter Mechanismus im Hirn“ darstellbar ist, der die Funktion hat, Individuen aneinander zu binden. ${ }^{15}$

11 Vgl. „Nächstenliebe“ in wikipedia (19.5.2016).

12 In der Literatur werden die Begriffe „Emotion“ und „Gefühl“ nicht einheitlich definiert. Ich bezeichne, dem üblichen Sprachgebrauch folgend, als Emotionen eher biosoziale „Hintergrundemotionen“ (wie Unbehagen-Wohlbehagen, Nervosität etc.) und universelle Basisemotionen (wie Trauer, Freude, Furcht, Ärger); als Gefühle die sozialen, kognitiv mit definierten Emotionen (wie Stolz, Scham, Liebe, Schuld usw.); vgl. dazu Damasio 1999.

13 „Leidenschaft, Gefühlsdrang: durch Vernunft schwer oder gar nicht bezwingliche Neigung“; Der Sprach-Brockhaus 1958, S. 397.

14 Vgl. dazu vor allem die Arbeiten von Thomas Scheff (1990).

15 Andreas Bartels, „Liebe im Blut“, The European. Das Debatten-Magazin, 25.4.2014 (1.5.2016). 
Liebesbezogene Erfahrungen in der Kindheit können genetische Folgen haben; Kinder, die wenig Liebe erfahren, haben später weniger Liebeshormone (etwa Oxytocin) im Blut. ${ }^{16}$ Diese Hormone fördern aber auch sozialen Ausschluss, Fremdenfeindlichkeit, Rassismus, weil der Liebesmechanismus mit der Stärkung unseres Gefühls der Zugehörigkeit zu einer bestimmten Gruppe auch Distanzierung von anderen erzeugt. Aus einer evolutionären Sicht ist Liebe oder Bindung daher ,[...] der Schlüssel zur Existenz von Arten wie dem Menschen, weil das Überleben ihrer Babys völlig von elterlicher Fürsorge abhängt, die nur durch Liebe möglich wird. ${ }^{\text {"17 }}$ Aus der Sicht der Neurobiologie sind Emotionen nicht (nur) eine das rationale Handeln begrenzende Rahmenbedingungen, sondern es gilt auch der umgekehrte Zusammenhang: ,[...] Rationalität ist eingebettet in die affektiv-emotionale Grundstruktur des Verhaltens; das limbische System entscheidet, ob, wann und in welchem Maße Verstand und Vernunft zum Einsatz kommen [...]" (Roth 2003, S. 560).

Liebe als ,verfeinertes, entwicklungsfähiges “ Gefühl. ${ }^{18}$ Wenn man von der biologischen Basis von Liebesbeziehungen ausgeht, ist eine breite Palette von Empfindungen relevant - von der ästhetisch-physischen Attraktion - mit ihren zwei Teilaspekten des Wohlgefallens am Aussehen des Partners und der erotisch-sexuellen Attraktion - bis hin zur Attraktion durch den Körpergeruch des Partners; dieser gibt Hinweise auf die Verträglichkeit der Immunsysteme der potentiellen Partner. ${ }^{19}$ Diese spielt die zentrale Rolle in der romantischen Liebe zwischen Mann und Frau - dem Paradefall einer Liebesbeziehung überhaupt. Vor allem beim Sich-Verlieben ist sie zentral; sie bleibt jedoch im Laufe der gesamten Beziehung relevant. Die englische Soziologin Catherine Hakim (2011) hat die Bedeutung der erotisch-sexuellen Ausstrahlung weit über Partnerbeziehungen hinaus überzeugend nachgewiesen. Interessant und wieder ein Hinweis auf den intellektualistisch-idealistischen Bias der soziologischen Forschung zu Liebe ist, dass dieser Aspekt des Aussehens kaum diskutiert wird; insofern setzt die heutige Forschung die Vernachlässigung des Körpers bei den soziologischen Klassikern (Gugutzer 2005, S. 24f.) fort. Es dominieren - genauso wie in den eingangs dargestellten soziologischen Handbüchern - eher problematische Aspekte wie Kontrolle des Körpers (Foucault), klassenspezifische Prägungen des körperlichen Habitus (Bourdieu) und natürlich geschlechtsspezifische Zuschreibungen an männliche und weibliche Körper (vgl. z.B. Shilling 2005).

Die erotisch-sexuelle Beziehung ist schon an sich viel mehr als nur etwas triebhaft-Biologisches. Der Biologe und Sexualtherapeut Kurt Loewit (1992, S. 72) verwendet in diesem Zusammenhang den Begriff Sprache der Sexualität: Er schreibt: Sexualität ist beim Menschen „,...]

16 Bartels, Liebe im Blut (s. FN 13); vgl. auch Roth (2003, S. 372).

17 Bartels, Liebe im Blut (s. FN 13); vgl. auch Hüther (2012, S. 13).

18 Die Begriffe „Emotion“ und „Gefühl“ werden in der Literatur nicht eindeutig voneinander abgegrenzt. Ich verwende hier den Begriff Emotion eher für die biologisch fundierte Empfindung der Liebe, den Begriff Gefühl für Liebe als ein stärker kognitives Phänomen, das auch soziokulturell definiert ist.

19 Vgl. dazu „Der betörende Duft des Immunsystems“, Die Welt (online. 2.10.2008), (abgerufen 5.6.2016). 
keine isolierte Lustquelle für sich, kein eigenes Kapitel, eben (nur) die notwendige Befriedigung des Geschlechtstriebes [...]; zu menschlicher Erotik und Sexualität gehören auch Erfahrungen und Kommunikationsweisen wie ,[...] aufeinander eingehen durch Sprechen und Hören, [...] sich mit den Augen ineinander versenken, sich im Händedruck, mit offenen Armen oder im Kuß begegnen, annehmen öffnen, miteinander verbunden sei $[\ldots]^{\text {“20 }}$ Vollkommen vereinbar mit dem Weber'schen Ansatz ist auch seine These, dass über die sexuelle Zufriedenheit nicht die Anzahl und Art sexueller Begegnungen entscheidet, sondern die Bedeutung, der Sinn, der ihnen beigemessen wird. Für die Philosophin Martha Nussbaum (2002, S. 193) ist sexuelles Begehren sowohl ein Trieb wie ein intentionales Phänomen, das sich auf ein bestimmtes Objekt bezieht und dies als begehrenswert definiert - was großteils ,im Kopf“ passiert. So ist es plausibel, dass eine gute Partnerbeziehung ihrerseits die sexuelle Empfänglichkeit erhöht (Clark and Wallin 1965). Die wirkliche sexuelle Revolution unserer Zeit besteht, wie der Soziologe Andrew Greeley (1977) in der ,[...] sehr modernen und sehr jungen Idee, dass Ehe, Freundschaft und sexuelle Erfüllung zusammen in ein- und derselben Beziehung bestehen können[...]“. ${ }^{21}$ Sexuelle Erfüllung ist ein zentraler Bestandteil der Beziehungsqualität in Ehen; er korreliert hoch mit positiver Bewertung von Liebe, Geborgenheit, Sicherheit und Kommunikation (Schulz 1983; Neto and da Conceicao Pinto 2013) 95\% aller sexuellen Interaktionen finden immer noch innerhalb der Ehe bzw. fester Partnerschaften statt. ${ }^{22}$ Randall Collins (2004) hat der sexuellen Interaktion in seiner Theorie der Interaktionsrituale, welche er als eine Hauptquelle für emotionale Energie bezeichnet, zu Recht einen zentralen Stellenwert eingeräumt. Er schreibt dazu:

„Sex is so far from normal bodily interaction that is it no surprise that it can produce the strongest of all forms of solidarity. In enormous contrast to ordinary bodily interaction, making love allows a person to tuch someone else's body; in high-intensity love-making, this may involve exploring, manipulating, doing things to every part of the other's body“" (Collins 2004, S. 234).

Aus dieser Sicht muss man sagen, dass sexueller Verkehr sogar eine engere soziale Beziehung als jene zwischen Mutter und Kind erzeugt, weil sie eine reziproke, emotionale und geistige Beziehung zwischen zwei erwachsenen, autonomen Personen darstellt.

20 Aus psychoanalytischer Sicht und gegen Freud's Verabsolutierung des Sexualtriebs wurde dieser Aspekt schon von Theodor Reik (1965) klar herausgearbeitet. Möglicherweise zu relativieren ist er allerdings im Zeitalter der vollen sexuellen „Befreiung“ mit häufigen seriellen Sexualbeziehungen mit wechselnden Partnerinnen und Partnern (Illousz 2011, S. 195).

21 Hier zitiert nach Loewit 1992, S. 69.

22 Interview mit dem Sexualforscher Volkmar Sigusch in der Süddeutschen Zeitung; vgl. http://sz-magazin.sueddeutsche.de/texte/anzeigen/43140/Ich-bin-in-Rage-angesichts-unserer-Sexualkultur (13.6.2016). 
Diese Einsichten scheinen in soziologischen Abhandlungen zur Bedeutung der Sexualität in Partnerbeziehungen vielfach zu fehlen. So schrieb etwa Helmut Schelsky (1955) in seiner Soziologie der Sexualität, die Ehe sei primär keine sexuelle Institution, weil der Geschlechtstrieb nicht die Dauerhaftigkeit der Ehe erkläre; es sei nur die Familienhaftigkeit - die Notwendigkeit einer Erweiterung der Mutter-Kind Beziehung durch einen Vater zur Sicherung der Kinderaufzucht - welche zur Institutionalisierung der Ehe als Regulierung des Geschlechtstriebs geführt habe; demzufolge definiert er Ehe und Familie als eine primär für die Fürsorge der Kinder etablierte „ökonomische Gemeinschaft“ (Schelsky 1955, S. 29). Eine Ausblendung der Erotik erfolgt auch in jenen feministischen Theorien (etwa jener von Judith Butler 1990) die einen „sexgereinigter“ Gender-Begriff verwenden (Reiche 1997). In solchen Sichtweisen werden die vielfältigen sozialen Funktionen, welche mit der erotisch-sexuellen Partnerbeziehung und mit der Familie verknüpft sind, offenkundig übersehen. Aus dieser Sicht muss es auch rätselhaft bleiben, dass die sexuelle Treue eine Grundnorm jeder stabilen erotisch-sexuellen Partnerschaft darstellt, die von einer überwältigenden Mehrheit der Bevölkerung anerkannt wird. ${ }^{23}$ Diese Norm gilt auch dann, wenn ein Ehepaar sich keine Kinder wünscht oder keine mehr bekommen kann. Es ist bezeichnend, dass dieses so wichtige Phänomen der ehelichen Treue im Buch von Schelsky keine wesentliche Rolle spielt. ${ }^{24}$

Der Handlungs- und Erfahrungsbezug

Wenn man von Max Weber ausgeht, sind nicht Gefühle, sondern soziales Handeln und soziale Beziehungen das Objekt der Soziologie. Man kann daher Liebe, oder besser: lieben, als eine Form von affektuellem oder emotionalem Handeln betrachten. Diese Sichtweise hat auch der Psychoanalytiker Erich Fromm in einem weit verbreiteten Büchlein vertreten:

„Liebe ist eine Aktivität und kein passiver Affekt. Sie ist etwas, das man in sich selbst entwickelt, nicht etwas, dem man verfällt. Ganz allgemein kann man den aktiven Charakter der Liebe so beschreiben, dass man sagt, sie ist in erster Linie ein Geben und nicht ein Empfangen.“ (Fromm 1995, S. 42)

Mit diesem Aspekt gelangen wir ins Zentrum einer soziologischen Betrachtung der Liebe aus wirklichkeitswissenschaftlicher Perspektive. Wir können hier wiederum mehrere Teilaspekte unterscheiden.

23 Nach den Ergebnissen des Sozialen Survey Österreich betrachteten 1986 und 1993 94\% der Befragten sexuelle Treue als wichtige Basis der Ehe, 2003 waren es 96\% (Haller 2005, S. 519). Aufgrund dieser einseitigen Verteilung hat ein Kollege in dieser kontinuierlich wiederholt durchgeführten sozialwissenschaftlichen Umfrage vorgeschlagen, die Frage nach der sexuellen Treue überhaupt weg zu lassen.

24 Jedenfalls taucht es nicht im Stichwortverzeichnis auf. 
Die körperliche und physische Nähe (Intimität): Geliebte möchten sich körperlich-physisch nahe sein, Zeit füreinander haben und verbringen, zusammen wohnen und essen, gemeinsame alltägliche und außeralltägliche Erfahrungen machen. Erfolgt dies kontinuierlich über längere Zeit, kann Liebe entstehen und/ oder sich vertiefen. Dieser Aspekt ist bei zwei Formen von Liebesbeziehungen höchst relevant. Zum einen bei der Mutterliebe: Schon die Erfahrung des engst möglichen, totalen körperlichen Beisammenseins zwischen Mutter und Kind im Laufe der neunmonatigen Schwangerschaft erzeugt in der Regel eine Bindung, der keine andere gleichkommen kann; durch die intensive Ernährung und Pflege des Babys und Kleinkindes in den ersten Lebensmonaten und -jahren wird diese Erfahrung noch vertieft. Zum anderen ist dieser Aspekt essentiell bei der romantischen Liebe: historisch und in manchen Kulturen wurde Liebe oft mit erotisch-sexueller Attraktion und Beziehung gleichgesetzt. Sie schaffen die engste Vertrautheit zwischen zwei Personen, die man sich vorstellen kann; Liebende teilen „Geheimnisse", die sie keinem Außenstehenden mitteilen. In der Psychologie wird dieser Aspekt mit dem Begriff der Intimität bezeichnet; er wird - neben Leidenschaft und Bindung - als einer der drei Eckpunkte des triangle of love gesehen (Sternberg 1988).

Der Raumbezug und die Zeitkomponente sind auch in Liebesbeziehungen von Erwachsenen von zentraler Bedeutung. Der in der heutigen, mobilen Zeit häufig gehörte Spruch: „Ich kann jemanden genauso lieben, wenn ich nur selten mit ihm beisammen bin“, scheint mir eher irreführend zu sein. Anthony Giddens hat die dahinterstehende Idee theoretisch „begründet“, indem er argumentierte, eine ...

„[...] reine moderne Paarbeziehung $[\ldots]^{\circ(}$ (pure relationship) sei jene, ,[...] in der alle externen Kriterien bedeutungslos geworden sind: die Beziehung existiert nur wegen der Gratifikationen, die sie verschafft. In einer solchen reinen Beziehung entsteht Vertrauen nur durch einen Prozess wechselseitiger Öffnung (disclosure)“ (Giddens 1991, S.6; Übersetzung MH).

Elisabeth Beck-Gernsheim (1983, S. 331) sieht diese Art von Beziehung, bei der im Mittelpunkt der Erwartungen an die Ehe die eigene Person, das persönliche Liebesglück, steht im Vormarsch. Giddens' ${ }^{6}$ Idee stellt zum Teil eine Wiederholung einer idealisierenden Vorstellung von der „romantischen Liebe“ dar; sie wurde kritisiert mit dem Argument, dass sie der Realität der faktischen Ungleichheit in vielen Paarbeziehungen widerspreche (Jamieson 1999). ${ }^{25}$ Im Grunde stellt sie eigentlich eine kosten-nutzenorientierte Perspektive dar, die weder den Eigenwert von sozialem Handeln noch die Bedeutung von ethisch-normativen Aspekten in engen Paarbeziehungen erkennt. Man könnte hier eine Feststellung des Philosophen Robert No-

$25 \mathrm{Vgl}$. auch „How viable is Giddens’ concept of the pure relationship. Evaluate his view of intimacy” http://www. essay.uk.com/free-essays/sociology/giddens-concept.php (1.5.2016). 
zick (1993, S. 128) anführen, die er im Zusammenhang mit der Frage was Glück sei, gemacht hat: „Ein Leben kann nicht einfach glücklich sein, ohne sonst etwas Wertvolles zu enthalten. Das Glück kommt huckepack auf anderen Dingen, die korrekt als positiv bewertet werden.“ Tatsächlich zeigen Studien, dass die Häufigkeit und Vielfalt der Aktivitäten, welche ein Paar oder eine Familie gemeinsam regelmäßig unternimmt, positive Auswirkungen hat. So hat das regelmäßige gemeinsame Einnehmen von Mahlzeiten positive Effekte für die Gesundheit von Kindern ${ }^{26}$ und die emotionale Stabilität von Teenagern. ${ }^{27}$ Auf diese soziale Bedeutung von gemeinsamen Mahlzeiten hat schon Georg Simmel (1997) hingewiesen (zit. in Shilling 2005, S. 151f.). Eine einfache Definition einer Liebes- oder Freundschaftsbeziehung könnte lauten: Ein echter Freund/Freundin ist jemand, mit dem oder der man gerne beisammen ist und der/die auch (fast) jederzeit dazu bereit ist. Hier ist ein Ergebnis aus einem australischen Zeitbudget-Survey relevant (Goodin et al. 2005): das häufige Klagen über Zeitmangel entsteht dadurch, dass die Menschen viel mehr Tätigkeiten ausüben als für ihr Einkommen und ihre Reproduktion nötig wären; diese „Zeitdruck-Illusion“ entsteht, wenn sie glauben, sie seien zu diesen Tätigkeiten gezwungen. Bezeichnenderweise ist der Zeitdruck am höchsten bei Personen in DoppelverdienerHaushalten, dagegen gering unter Alleinerzieher/innen. Für Ehefrauen, deren Gatten infolge langer Arbeitszeiten und vieler außerhäuslicher Hobbies selten zu Hause sind, ist Langeweile eine typische Erfahrung (Klapp 1986, S. 11).

Eine weitere Situation, in welcher man die Bedeutung des räumlich-physischen Zusammenlebens erkennt, ist jene von Ehepaaren mit Kindern, die sich scheiden lassen. Für die USA wurde gezeigt, dass Frauen signifikant häufiger die Scheidung beantragen, wenn sie erwarten können, dass ihnen der Scheidungsrichter die Obsorge über die Kinder zuschreiben wird. ${ }^{28}$ In ihrem Buch Der gebrauchte Mann beschreibt Karin Jäckel (2000) zahlreiche Fälle von Ehescheidungen in Deutschland, bei denen die Männer nach Bekanntgabe des Scheidungswunsches ihrer Frau aus allen Wolken fielen und dass sich daraus oft dramatische Konsequenzen (bis hin zum Selbstmord) zogen; für diese Frauen war das Motiv der Eheschließung von vornherein nur gewesen, ein legitimes Kind zu bekommen und mit diesem (ohne Mann) zusammen zu leben.

Einsamkeit als Auslöser von Liebessehnsucht und Bereitschaft zum Sich-Verlieben - die situative Komponente: Die einmalige, intellektuell schwer nachvollziehbare Erfahrung des Sich-Verliebens ist wohl einer der Gründe für die Unterbelichtung des Themas Liebe in der Soziologie. Der italienische Soziologe Francesco Alberoni (2000) hat die These entwickelt, dass das Sich-Verlieben kein Zufallsprozess ist, sondern dass wir in ganz bestimmten sozialen

26 Fiese et al. (2011) fanden, dass sich gemeinsame Familienmahlzeiten positiv auf die Heilung von Asthma bei Kindern auswirkten; besonders förderlich waren freundliche Gespräche und reger Gedankenaustausch während der Mahlzeiten stattfand und elektronische Geräte ausgeschaltet waren.

27 „Familienmahlzeiten fördern emotionale Stabilität von Teenagern“, erziehungstrends.de, 18.4.2013; vgl. http:// www.erziehungstrends.net/Familienmahlzeiten (2.5.2016).

28 Vgl. Brinig and Allen (2000). 
Situationen und Lagen dafür prädestiniert sind uns zu verlieben oder zumindest uns dringlich wünschen, eine Liebesbeziehung zu erfahren, nämlich dann, wenn wir ein starkes Gefühl der Einsamkeit empfinden. Die Liebe eröffnet einen Ausweg aus dem Gefühl des Alleinseins, denn durch eine tiefe Liebeserfahrung erhalte die Welt eine ganz neue Ausrichtung, unser Blick auf sie wird viel positiver. Die Soziologin Rosantonietta Scramaglia (2000) hat daraus die weitere These abgeleitet, dass es im Laufe des Lebens immer wieder Phasen geben müsse, in welchen man sich verliebe und sie konnte diese durch retrospektive Befragungen bestätigen; die erste Erfahrung des Sich-Verliebens erfolgte bereits im Kindergarten - also der Phase, in der das Kind erstmals das behütete Elternhaus für längere Perioden verlässt; weitere erfolgten im Alter von 5 bis 6,10 bis 11 und mit 14 Jahren, immer im Zusammenhang mit kritischen Übergängen in eine neue bzw. andere Ausbildungsstätte.

Die kognitive Komponente der Liebe

Liebe beinhaltet auch eine kognitive Komponente. Ich muss die geliebte Person kennen und verstehen, damit ich sie „wirklich“ lieben kann. Dieser Aspekt besitzt sehr große Bedeutung in einer entwickelten Liebesbeziehung (vgl. dazu ausführlich Lenz 2003, S. 167-248). Peter Berger und Hansfried Kellner (1965) haben argumentiert, dass die Ehe heute eine zentrale Institution zum Aufbau einer gemeinsamen Wirklichkeitsdefinition der Partner darstelle; im Kontext dieser werde auch die individuelle Identität der Partner stabilisiert. Die Bedeutung der kognitiven Komponente im Prozess des Sich-Verliebens hat der Schriftsteller Stendhal in einem Buch Über die Liebe (1966) sehr schön beschrieben. Er berichtet Folgendes: Wenn man im Innern des Salzburger Bergwerks einen Zweig in den Höhlensee wirft und ihn nach einigen Tagen wieder herausnimmt, ist er über und über voll von wunderschönen Kristallen. Ähnlich entwickelt sich laut Stendhal die Vorstellung von einer Person, in die man verliebt ist, die man aber einige Zeit nicht sieht: Sie wird von der Phantasie immer schöner und schöner ausgemalt. Trifft man die Person wieder, lässt sich dieses Bild natürlich kaum halten. Auch die Erfahrung, dass frisch Verliebte sich ungeheuer viel zu erzählen haben, mag damit zusammenhängen; man versucht sich eben so weit als möglich kennen zu lernen. Dazu dienen vor allem biographische Erzählungen über die bisherigen Lebensgeschichten beider Partner (Lenz 2003, S. 199). Das Zusammenleben lange (und einigermaßen gut) verheirateter Paare mag auch deswegen von ihnen als gut empfunden werden, weil sie über viele alltägliche Dinge kaum mehr viele Worte verlieren müssen, und weil sie auch die Bedürfnisse, Wünsche und Vorlieben ihrer Partner sehr gut kennen. Das ist aber natürlich auch umso besser möglich, je mehr sie beisammen sind, miteinander kommunizieren und gemeinsame Erfahrungen machten. So beinhalten starke Gefühle für einen anderen Menschen auch Überzeugungen, und zwar vor allem darüber, welche Ereignisse im Zusammenhang mit ihm stattgefunden haben (Nussbaum 2002, S. 174). 
Die ethisch-normative Komponente der Liebe

Wenn ich jemanden liebe, fühle ich mich auch verpflichtet ihn im Alltag zu unterstützen, ihn zu trösten, ihm in einem Notfall bedingungslos zu helfen. Dieses Helfen ist keine lästige, von außen oktroyierte (wenngleich sozial und kulturell durchaus gestützte) Norm, sondern etwas, was man gerne tut. Hier gilt paradigmatisch die These von Friedrich Schiller, dass die Lust am Schönen, am Rührenden, am Erhabenen an der Liebe auch unsere moralischen Gefühle stärkt (Schiller 1962). Wenn es dem Geliebten schlecht geht, geht es auch mir schlecht. Eine sehr einfache, meiner Meinung nach treffende Definition von Liebe (und Haß) hat der Philosoph David Hume (1978, S. 101) gegeben: „Liebe ist gar nichts anderes, als der Wunsch, dass eine andere Person glücklich, und Haß der Wunsch, dass sie unglücklich sein möge." Wichtig in diesem Zusammenhang ist der Begriff des commitment, der Verpflichtung, mit jemand anderem eine intensive, dauerhafte Liebesbeziehung aufbauen, den Partner in jeder Hinsicht und Lebenslage unterstützen zu wollen (Kelley 1993). Liebe ist hier sehr nahe verwandt dem Begriff der Sympathie, der Fähigkeit, sich in das Leid, aber auch die Freude anderer einzufühlen. Bedeutende Autoren (Shaftesbury, D. Hume, A. Smith, A. Comte, H. Spencer) haben in der Sympathie die Grundlage für die Entstehung von ethisch-moralischem Handeln gesehen. Ja, Sympathie ist geradezu identisch mit den ersten Stadien des sich-Verliebens, wenn sie definiert wird als „[...] die scheinbar grundlose Zuneigung zu jemandem, das unbestimmte Gefühl der inneren Verwandtschaft mit jemandem. ${ }^{\text {" }}{ }^{29}$

Die Institutionalisierung von Liebesbeziehungen. Die Relevanz dieser ethisch-moralischen Komponente in Liebesbeziehungen wird offenkundig in der Tatsache, dass die Partnerbeziehung und die Eltern-Kind-Beziehung und die daraus entstehenden Gemeinschaften von Ehe und Familie in allen Gesellschaften öffentlich geregelt und normiert, also institutionalisiert werden. Der Hauptgrund dafür ist natürlich, dass durch diese Institutionen die Aufzucht der Kinder gesichert wird, die Gesellschaft daher hohes Interesse daran hat, dass dies in einer zuträglichen Weise geschieht. Die Institutionen von Ehe und Familie sind daher nicht in erster Linie eine Regulierung und Institutionalisierung „urwüchsiger“ sexueller Beziehungen Weber (1964, S. 275-279); vielmehr ,,...] kreuzen Vergemeinschaftsbeziehungen aller Art [...] die sexuellen und physiologischen Beziehungen [...]“; auch der Begriff der „Ehe“ ist nur durch Bezugnahme auf andere Einheiten als die der Aufzuchtsgemeinschaft von Mutter, Vater und Kindern zu verstehen. Allerdings kommt Weber über den Umweg der These, dass die Hausgemeinschaft auch die sexuellen Beziehungen zwischen ihren Mitgliedern regulieren müsse, letztlich doch wieder zur Sexualität als einer Triebkraft der Differenzierungsprozesse innerhalb der Hausge-

29 Meyers Großes Konversations-Lexikon 1909; siehe: http://www.zeno.org/Meyers-1905/A/Sympath\%C4\%ABe. vgl. auch „Sympathie“ in http://de.wikipedia.org/Sympathie. 
meinschaft (Radkau 2005, S. 43). ${ }^{30}$ Eine moderne, adäquate Institutionalisierung müsste vor allem auch geschlechtsspezifische Differenzen in der Bedeutung von Liebesbeziehungen in den Griff beachten, wie Eva Illousz (2011, S. 439) schreibt:

„Da Frauen noch immer nicht bereit sind, der Idee der romantischen Liebe abzuschwören, und da sie Männern auf einem offenen Feld begegnen, muß die Akkumulation sexuellen Kapitals diskutiert und hinterfragt werden, um neue Strategien zu ersinnen, wie man mit emotionalen Ungleichheiten umgeht und die umfassenderen sozialen und ethischen Ziele von Frauen verwirklicht.“

\section{Das Ideal der romantischen Liebe}

Um die Relevanz der wirklichkeitssoziologische Perspektive noch deutlicher zu machen, scheint es in diesem Zusammenhang angebracht, kurz das berühmte Ideal der romantischen Liebe zu diskutieren. Dies ist eine im Laufe des 17. und 18. Jahrhundert zuerst in der schöngeistigen Literatur in England und in der deutschen Romantik entwickelte, idealisierte Vorstellung der Partnerbeziehung, die als durchaus modern anzusehen ist. Wenn man sich fragt, welche Bedeutung Liebe und Ehe heute - im Zeitalter sinkender Heiratsraten, steigender Scheidungsraten usw. - noch besitzen, muss man sich mit diesem Ideal auseinandersetzen. Soziologisch relevant wird dieses Ideal, weil sich auch die Erwartungen und das Verhalten der Menschen in bestimmten Gesellschaften und Epochen daran orientieren (was nicht heißt: es immer befolgen).

Nach Karl Lenz (2003, S. 259) beinhaltet dieses Ideal sechs Elemente: (1) die Einheit von sexueller Leidenschaft und affektiver Zuneigung; (2) das Postulat der Einheit von Liebe und Ehe; (3) die Elternschaft als wesentlicher Teil der ehelichen Liebesbeziehung; (4) die Dauerhaftigkeit der Liebe und die Treue der Partner; (5) die Einbeziehung der Individualität der beiden Liebenden, insbesondere auch der Frau; (6) die Aufwertung der Liebe zum höchsten Gut im Leben, die Verheißung des höchsten Glücks durch sie. Alle diese Annahmen waren revolutionär, weil bis dahin erotisch-sexuelle Beziehungen keinen geistigen Eigenwert besaßen, Sexualität, Liebe und Ehe weitgehend getrennt waren, die Ehe vielfach eine ökonomische Versorgungsgemeinschaft darstellte usw. Es scheint mir nun unzureichend, dieses Ideal nur mit der Realität von heute zu konfrontieren, wie es meist geschieht (vgl. z.B. Lenz 2003, S. 283). Vielmehr muss man sich auch fragen, inwieweit diese Vorstellung als handlungsleitendes Ideal angesehen werden kann. Manche Autoren bestreiten dies. So sieht Luhmann in seinem Buch Liebe als Passion

30 Radkau ist an dieser Stelle allerdings zu widersprechen, wenn er meint, damit kehre bei Weber „,die aus der Hausgemeinschaft verbannte Natur“" wieder zurück; sexuelle Beziehungen zwischen Menschen sind eben mehr als nur „Natur“. 
diese nur als einen kulturellen Code, der den Menschen seit jener Zeit dazu diente, entsprechende Gefühle überhaupt erst zu entwickeln: „Liebe wird hier nicht, oder nur abglanzweise, als Gefühl behandelt, sondern als symbolischer Code, der darüber informiert, wie man ... erfolgreich kommunizieren kann. Der Code ermutigt, entsprechende Gefühle zu bilden. Ohne ihn würden die meisten ... gar nicht zu solchen Gefühlen finden.“(Luhmann 1982, S. 9). Richtig an diesen Ausführungen ist natürlich, dass auch die Emotion der Liebe - wie alle Emotionen -kognitive Komponenten hat und dadurch, dass junge Menschen Liebesromane, Ratgeberbücher für Partner- und Liebesbeziehungen lesen, durch diese beeinflusst werden. Die unabhängige, emotionale Basis der Liebe zu verneinen, widerspricht aber vollkommen einem wirklichkeitssoziologischen Ansatz und natürlich auch der neurobiologischen Sicht (Roth 2003).

$\mathrm{Zu}$ den vorhin genannten sechs Kriterien der „romantischen Liebe“ ist festzustellen: Kennzeichen 1, 5 und 6 (Verbindung von sexueller Beziehung und affektiver Zuneigung, Dauerhaftigkeit, Einbeziehung der Individualität beider Partner) kann man auch aufgrund der in Übersicht 1 angeführten Kriterien als wesentlich ansehen; nur zum Teil jedoch die Kriterien 2 und 3 (Verbindung zwischen Liebe und Ehe bzw. Liebe, Ehe und Elternschaft); überhaupt nicht Kriterium 6 (Liebe als wichtigste Sache im Leben). Eine tiefe Liebesbeziehung ist vorstellbar auch außerhalb der Ehe und ohne Kinder. Dies zu sehen ist besonders wichtig in Phasen abnehmender Heiratsraten und zunehmender Kinderlosigkeit. Nicht nur deshalb, sondern auch aufgrund der gestiegenen Lebenserwartung ist die Phase der ,nachelterlichen Gefährtenschaft“ (Rosenmayr 1986; Schmitt 2010) ist die Partner- bzw. Ehebeziehung heute grundsätzlich bedeutsamer geworden (Veenhoven 1983). Sie muss viel stärker als eigenständige Form der Beziehung untersucht werden, nicht nur unter dem Blickwinkel der Familie (Lenz 2003, S. 9ff.). Anderseits ist evident, dass im Ideal der romantischen Liebe einige wichtigen Aspekte einer Liebesbeziehung fehlen, nämlich der Aspekt der Intimität, des Aussehens bzw. der Attraktivität des Partners, der Aspekt des Zusammenlebens und der gemeinsamen Erfahrungen und schließlich der Aspekt der gegenseitigen Verpflichtung. Unter diesem Aspekt würde ich meinen, dass dieses Ideal weiterhin für all jene Menschen gültig ist, die nicht unter sehr depravierten Verhältnissen (wie manche Afroamerikanerinnen; vgl. Clarke 2011) leben müssen. Dies trifft selbst auf Afrika zu; romantische Liebesbeziehungen sind auch hier ein zentrales Thema von Romanen, Musik, Zeitungen und Magazinen - ein Faktum, das die westliche Ethnologie und Sozialforschung weitgehend übersehen hat, wenn sie Intimität in Afrika allenfalls als Sexualität identifizierte (Cole and Thomas 2009, S. 1-6). 


\section{Thesen zur Bedeutung und Zukunft von Liebesbeziehungen}

Es würde zu weit führen, an dieser Stelle noch ausführlich auf die Bedeutung der vorgestellten Überlegungen für den Wandel und die Zukunft von Liebesbeziehungen einzugehen. Mir scheint jedoch, dass sich daraus doch einige neue Perspektiven aus einer Reihe aktueller Probleme und Tendenzen ergeben. Nur beispielhaft seien einige davon angesprochen.

Wir haben gesehen, dass Liebe und Liebesbeziehungen im gesamten Lebenslauf eine wichtige Rolle spielen; von der Kindergartenzeit bis in das hohe Alter. In verschiedenen Lebensphasen treten unterschiedliche Formen von Liebesbeziehungen in den Vordergrund: In Kindheit und Jugend die Beziehung zwischen Eltern und Kindern, zwischen Geschwistern, die zwischen Freundinnen und Freunden in der peer group; im (jungen) Erwachsenenalter Verwandtschaftsund Freundschaftsbeziehungen (auch vermittelt über berufliche Kontakte); erotisch-sexuelle Partnerbeziehungen; im Alter Beziehungen zu einem Partner, zu (auch neuen) Freundinnen und Freunden; erwachsenen Kindern; zu Geschwister, falls vorhanden. Wichtig ist, dass in jeder Lebensphase einige solcher Beziehungen in einer sehr positiven Form bestehen; ist dies nicht der Fall, können sich schwere Lebenskrisen ergeben. Andererseits können solche neuen Beziehungen auch Defizite ausgleichen, welche in vorhergehenden Lebensphasen entstanden sind. ${ }^{31}$

Sieht man erotisch-sexuelle Erfüllung als zentrales Element der Liebe in einer Partnerbeziehung, die weit mehr umfasst als nur physische Aspekte (auch Zärtlichkeit, Kommunikation, usw.), so wird auch evident, dass eine tiefe erotisch-sexuelle Beziehung für die Partner- und Ehebeziehung sehr wichtig ist, wie Wolfgang Schulz und Florian Pichler (2005) anhand des Sozialen Survey Österreich gezeigt haben. Andererseits ist eine wenig befriedigende sexuelle Beziehung ein Indikator für Probleme in der Beziehung und oft ein Anlass für die Aufnahme sexueller Beziehungen zu einem anderen Partner; diese sind ihrerseits häufig die Ursache für Trennungen und Scheidungen - ein Aspekt, der heute vielfach bagatellisiert wird. Auch die Folgen der sexuellen Revolution für Partnerbeziehungen werden deutlicher. Im Zuge der Entwicklung effizienter Methoden der Empfängnisverhütung ist es möglich geworden, Sexualität und eine tiefergehende erotische Partnerbeziehung zu trennen; die stark verlängerte Phase der Jugend (früherer Beginn der Pubertät, späteres Eintreten in die volle Erwachsenenrolle) mag viele Jugendliche dann dazu führen, Partnerbeziehungen als grundsätzlich ,auf Zeit“ angelegt zu betrachten; die Häufigkeit von Scheidungen und Wiederverheiratungen erscheint dann nur als Fortsetzung dieses Musters.

„Reife“ Liebe, so habe ich argumentiert, entsteht vor allem durch kontinuierliches Zusammenleben und eine Vielzahl an gemeinsamen Aktivitäten (gemeinsames Essen, Wohnen, Freizeit und Urlaub verbringen usw.). Zwar hat die Ausdehnung der Freizeit potentiell zu einer starken Ausweitung der zeitlichen Möglichkeiten für gemeinsame Aktivitäten von Partnern ge-

31 Für empirische Evidenz vgl. Brockmann und Klein 2004. 
führt; auf der anderen Seite führt die Erwerbstätigkeit beider Partner, im Zusammenhang mit der Flexibilisierung der Arbeitszeit und steigender beruflich-geographischer Mobilität dazu, dass die gemeinsam verbrachte Zeit nicht unbedingt zunimmt. Die Frage ist auch, ob gemeinsames Fernsehen - die heute mit Abstand häufigste Freizeitbeschäftigung - wirklich als gemeinschaftliche „Aktivität“ anzusehen ist; sehr langes Fernsehen hängt jedenfalls mit geringerer Lebenszufriedenheit zusammen (Frey, Benesch and Stutzer 2007).

Echte und tiefe Liebe führt dazu, dass andere Aspekte in einer Partnerbeziehung oder Ehe (Arbeitsteilung im Haushalt, Einkommensverwendung, Machtverteilung) eine untergeordnete Rolle spielen bzw. Konflikte darüber ohne große Probleme gelöst werden können. Der wissenschaftliche Neuigkeitswert und praktische Nutzen der x-ten Studie darüber, wer im Haushalt das Geschirr abwäscht, den Müll wegbringt usw. ist sehr begrenzt. Ich kenne jedoch kaum Studien, welche die Stärke der Liebesbeziehung selber thematisieren und empirisch erfassen. ${ }^{32}$ Dabei ist evident, dass tiefe Liebe am meisten hilft Probleme in einer Beziehung zu bewältigen. Die Bewältigung dieser Probleme stärkt ihrerseits die Liebe (z.B. Pflege schwerbehinderter Kinder durch ihre Eltern; Pflege eines erkrankten Partners durch den anderen). Man könnte vermuten, dass die Vernachlässigung der Darstellung dieser positiven Aspekte von Partnerbeziehungen zugunsten des Herausstellens von Problemen mit ein Grund dafür ist, dass die Skepsis gegen das Eingehen einer Beziehung erhöht und damit auch ein Grund etwa für die niedrige Heiratsquote unter weiblichen Akademikerinnen ist.

Durch Enttäuschungen und Verletzungen kann eine Liebesbeziehung in Hass umschlagen. Dadurch können große Probleme bei Trennungen und Ehescheidungen entstehen, vor allem dann, wenn auch Kinder involviert sind (Preglau 1998). Wenn Ehescheidungen heute als etwas weitgehend Problemloses dargestellt werden, übersehen sie dieses wichtige Faktum. Es beruht seinerseits darauf, dass die meisten Ehescheidungen zwar juristisch, nicht aber sozial einvernehmlich ablaufen; in der Realität ist es meist ein Partner, der die Scheidung betrieben hat, während der andere sie nicht unbedingt oder überhaupt nicht wollte (Wallerstein und Blakeslee 1989). Hier kommt auch der ethisch-normative Aspekt ins Spiel. Während die Ehe als Institution immer mehr an Bedeutung verliert - indiziert auch durch die Zunahme von Partnerschaften ohne Trauschein - verschwindet die persönliche Verantwortung von Partnern gegeneinander, auch nach einer Trennung, keineswegs. Eine Soziologie als Wirklichkeitswissenschaft sollte wohl - ohne ins Moralisieren zu verfallen - zu einem stärkeren Bewusstsein dieser Tatsache beitragen.

Zusammenfassend würde ich sagen, dass es heute Tendenzen gibt, welche die Entwicklung von Liebesbeziehungen in allen unterschiedlichen Formen erschweren, aber auch solche, wel-

32 Ein Oberbegriff, unter welchem dies z.T. geschieht, ist der der „Beziehungsqualität“ (vgl. Dette-Hagenmeyer und Reichle 2015). Eine Skala zur Messung leidenschaftlicher Liebe haben Elaine Hatfield und Susan Sprecher (1986) entwickelt; vgl. auch Hatfield, Bensman and Rapson 2012. 
che sie erleichtern und fördern. Die letzteren scheinen jedoch - trotz vielfältiger Krisenerscheinungen - deutlich zu überwiegen. So zeigt auch die Lebenszufriedenheit der Österreicherinnen und Österreicher eine deutlich zunehmende Tendenz im Zeitverlauf. ${ }^{33}$ Das Verschwimmen der Grenzen zwischen Öffentlichkeit und Privatheit in Fernsehen und sozialen Medien, Kommerzialisierung und Konsumorientierung, Instrumentalisierung von sexuellen Beziehungen, zunehmende Mobilität erschweren den Aufbau tiefer, längerfristiger Beziehungen; die steigende Bildung, insbesondere von Frauen, sowie deren wirtschaftliche Selbständigkeit, der Abbau traditioneller Geschlechtsrollenerwartungen erhöhen dagegen die Chancen für gleichberechtigte Beziehungen; verbesserte Lebensbedingungen und höhere Lebenserwartung verlängern die Dauer von Beziehungen; auch die Verkürzung der Arbeitszeiten kann sozialen Beziehungen zu gute kommen; die enorm erleichterte Kommunikation und Mobilität ermöglicht die Aufrechterhaltung von Beziehungen auch über weite Distanzen.

\section{Literatur}

Alberoni, Francesco. 2000. Historisierung und Punkte ohne Wiederkehr. Grenzüberwindung und Grenzziehung im kollektiven Projekt eines verliebten Paares. In: Grenzen und Grenzüberschreitungen der Liebe, hrsg. Kornelia Hahn und Günter Burkart, 11-26. Opladen: Leske + Budrich.

Beck-Gernsheim, Elisabeth. 1983. Vom ,Dasein für andere` zum Anspruch auf ein Stück, eigenes Leben`. Individualisierungsprozesse im weiblichen Lebenszusammenhang, Soziale Welt 34: 307-340.

Beck, Ulrich und Elisabeth Beck-Gernsheim. 1990. Das ganz normale Chaos der Liebe. Frankfurt am Main: Suhrkamp.

Berger, Peter und Hansfried Kellner. 1965. Die Ehe und die Konstruktion der Wirklichkeit. Eine Abhandlung zur Mikrosoziologie des Wissens. Soziale Welt 16: 220-235.

Brinig, Margaret F. and Douglas W. Allen. 2000. 'These boots are made for walking': Why most divorce filers are women. American Law and Economics Review V2N1: 126-169.

Brockmann, Hilke and Thomas Klein. 2004. Love and death in Germany. The marital biography and its effect on mortality. Journal of Marriage and the Family 66: 567-581.

Burkart, Günter. 2000. Arbeit und Liebe. Über die Macht der Liebe und die Arbeit an der Partnerschaft. In: Grenzen und Grenzüberschreitungen der Liebe, hrsg. Kornelia Hahn und Günter Burkart, 165-198. Opladen: Leske + Budrich.

Butler, Judith. 1990. Das Unbehagen der Geschlechter. Frankfurt am Main: Suhrkamp.

33 Nach den Ergebnissen des Sozialen Survey Österreich (repräsentative sozialwissenschaftliche Umfragen) waren 1984 67\% der Österreicherinnen und Österreicher mit ihrem Leben insgesamt ziemlich oder sehr zufrieden, 1986 76\%, $199378 \%$ und 2003 81\%; der Anteil der sehr Zufriedenen entwickelte sich folgendermaßen: 23\%, 27\%, 30\%, $35 \%$ (Schulz und Pichler 2005, S. 80). 
Clark, Alexander J. and Paul Wallin. 1965. Women's sexual responsiveness and the duration and quality of their marriages. American Journal of Sociology LXXI: 187-196.

Clarke, Averill. 2011. Inequalities in Love. College-Educated Black Women and the Barriers to Romance and Family. Durham, NC: Duke University Press.

Cole, Jennifer and Lynn M. Thomas. eds. 2009. Love in Africa. Chicago/ London: The University of Chicago Press.

Collins, Randall. 2004. Interaction Ritual Chains. Princeton/ Oxford: Princeton University Press.

Connell, Raewyn and Rebecca Pearse. 2015. Gender in World Perspective. Cambridge: Polity Press.

Dalai Lama. 2016. Die Liebe. Quelle des Glücks. Freiburg/Basel/Wien: Herder.

Damasio, Antonio. 1999. Ich fühle, also bin ich. Die Entschlüsselung des Bewusstseins. München: List.

Dette-Hagenmeyer, Dorothea E. und Barbara Reichle. 2015. Beziehungsqualität, partnerschaftliche Interaktion und Auswirkungen auf angehörige Kinder. In: Handbuch Familiensoziologie, hrsg. Paul B. Hill und Johannes Kopp, 437-461. Wiesbaden: Springer Fachmedien Wiesbaden.

Durkheim, Emile 1987. Der Selbstmord. Frankfurt am Main: Suhrkamp (zuerst 1897)

Fiese, Barbara H.; Marica A. Winter and Joanna C. Botti. 2011. The ABCs of Family Mealtimes: Observational Lessons for Promoting Healthy Outcomes for Children With Persistent Asthma. Child Development 82: 133-145.

Firestone, Shulamit. 1975. Frauenbefreiung und sexuelle Revolution. Frankfurt am Main: Fischer Taschenbuch

Flam, Helena. 2002. Soziologie der Emotionen. Eine Einführung. Konstanz: UVK

Frey, Bruno S.; Christine Benesch and Alois Stutzer. 2007. Does watching TV make us happy? Journal of Economic Psychology. 28: 283-313.

Fromm, Erich. 1995. Die Kunst des Liebens. München: dtv (zuerst 1956).

Giddens Anthony. 1991. Modernity and Self-Identity. Self and Society in Late Modern Age. Cambridge: Polity Press.

Goodin, Robert E. et al. 2005. The time-pressure illusion: Discretionary time vs. free time. Social Indicators Research 73: 43-70.

Goode, William J. 1974. The theoretical importance of love. In: The Family. Its Structures \& Functions, hsrg. Rose Laub Coser, 143-156. London/Basingstoke: Macmillan.

Greeley, Andrew. 1977. Erotische Kultur. Graz/Wien/Köln: Styria.

Gugutzer, Robert. 2005. Soziologie des Körpers. Bielefeld: transcript.

Habermas, Tilman. 1999. Geliebte Objekte. Symbole und Instrumente der Identitätsbildung. Frankfurt am Main: Suhrkamp.

Hahn, Kornelia und Günter Burkart. hrsg. 2000. Grenzen und Grenzüberschreitungen der Liebe. Studien zur Soziologie intimer Beziehungen II. Opladen: Leske + Budrich.

Hakim, Catherine. 2011. Erotisches Kapital. Das Geheimnis erfolgreicher Menschen. Frankfurt am Main/ New York: Campus. 
Haller, Max. 1980. Das Heiratsinserat. Ein Beispiel für die Interdependenz von Statushierarchie und Heiratsmarkt. Österreichische Zeitschrift für Soziologie 5:62-65

Haller, Max 1983. Theorie der Klassenbildung und sozialen Schichtung. Frankfurt am Main/ New York: Campus.

Haller, Max. 2005. Auf dem Weg zur mündigen Gesellschaft? Wertwandel in Österreich 1986-2003. In: Österreich zur Jahrhundertwende. Gesellschaftliche Werthaltungen und Lebensqualität 1986-2004, hrsg. Wolfgang Schulz; Max Haller und Alfred Grausgruber, 33-73. Wiesbaden: VS Verlag für Sozialwissenschaften.

Haller, Max. 2008. Die österreichische Gesellschaft. Sozialstruktur und sozialer Wandel. Frankfurt am Main/New York: Campus.

Haller, Max. 2013. Geschwister-Beziehungen. Eine wichtige soziale Ressource in modernen Gesellschaften. soziologie heute, Jg. 6, Heft 27: 12-14.

Hatfield, Elaine and Susan Sprecher. 1986. Measuring passionate love in intimate relationships. Journal of Adolescence 9: 383-410.

Hatfied, Elaine; Lisamaria Bensman and Richard L. Rapson. 2012. A brief history of social scientists“ attempt to measure passionate love. Journal of Social and Personal Relationships 29: 143-164.

Heidbrink, Horst. 2007. Freundschaftsbeziehungen. Journal für Psychologie 15. http://www.journal-fuerpsychologie.de/index.php/jfp/article/view/122/109

Hill, Paul Bernhard und Johannes Kopp. hrsg. 2015. Handbuch Familiensoziologie. Wiesbaden: Springer VS.

Hume, David. 1978. Ein Traktat über die menschliche Natur. Über die Affekte. Über Moral. Hamburg: F. Meiner.

Hüther, Gerald. 2012. Die Evolution der Liebe. Was Darwin bereits ahnte und die Darwinisten nicht wahrhaben wollen. Göttingen: Vandenhoeck \& Ruprecht.

Illousz, Eva 2011), Warum Liebe weht tut. Eine soziologische Erklärung. Frankfurt am Main: Suhrkamp. Jäckel, Karin. 2000. Der gebrauchte Mann. München: Deutscher Taschenbuch Verlag.

Jamieson, Lynn. 1999. Intimacy transformed: A critical look at the 'pure relationship'. Sociology 33 : 477-494.

Kelley, Harold H. 1993. Love and commitment. In: Close Relationships, eds. Harold H. Kelley et al., 265314. New York/San Francisco: W.H. Freeman and Company.

Klapp, Orrin E. 1986. Overload and Boredom. Essays on the Quality of Life in the Information Society. New York: Greenwood Press.

Klein, Thomas, Hrsg. 2001. Partnerwahl und Heiratsmuster. Sozialstrukturelle Voraussetzungen der Liebe. Opladen: Leske + Budrich.

Lenz, Karl. 2003. Soziologie der Zweierbeziehung. Eine Einführung. Opladen: Westdeutscher Verlag. Loewit, Kurt. 1992. Die Sprache der Sexualität. Frankfurt am Main: Fischer Taschenbuch Verlag. Luhmann, Niklas. 1982. Liebe als Passion. Zur Codierung von Intimität. Frankfurt am Main: Suhrkamp. 
Nave-Herz, Rosemarie und Manfred Markefka. hrsg. 1989. Handbuch der Familien- und Jugendforschung, Bd. 1: Familienforschung. Neuwied/Frankfurt am Main: Luchterhand.

Neto, Felix and Maria da Conceicao Pinto. 2013. The satisfaction with sex life across the adult life span. Social Indicators Research 114: 767-784.

Nozick, Robert. 1993. Vom richtigen, guten und glücklichen Leben. München: dtv.

Nussbaum, Martha. 2002. Konstruktion der Liebe, des Begehrens und der Fürsorge. Stuttgart: Reclam.

Peterson, Gary W./ Kevin R. Bush, eds. 2013. Handbook of Marriage and the Family. New York etc.: Springer.

Preglau, Max. 1998. 'Postmodernisierung' des Selbst? Versuch einer theoretischen und empirischen Annäherung. In: Postmodernes Österreich? hrsg. Max Preglau und Rudolf Richter, 353-371. Wien: Signum Verlag.

Radkau, Joachim. 2005. Max Weber. Die Leidenschaft des Denkens. München: Carl Hanser.

Reiche, Reimut. 1997. Gender ohne Sex. Geschichte, Funktion und Funktionswandel des Begriffs 'Gender'. Psyche 51: 926-958.

Reik, Theodor. 1965 [1945]. Geschlecht und Liebe. München: Kindler.

Roth, Gerhard. 2003. Fühlen, Denken, Handeln. Wie das Gehirn unser Verhalten steuert. Frankfurt am Main: Suhrkamp.

Rosenmayr, Leopol. 1986. Über Familie in den Strukturumbrüchen heute. Archiv für Wissen und Praxis der sozialen Arbeit, Heft 2-4: 48-81.

Scheff, Thomas. 1990. Microsociology. Discourse, Emotion and Social Structure, Chicago/London: The University of Chicago Press.

Scheff, Thomas 2011. What's Love Got to do with it? Emotions and Relationships in Popular Songs. Boulder/London: Paradigm Publishers.

Schelsky, Helmut. 1955. Soziologie der Sexualität. Über die Beziehungen zwischen Geschlecht, Moral und Gesellschaft. Hamburg: Rowohlt.

Schiller, Friedrich. 1962. Über den Grund des Vergnügens an tragischen Gegenständen. In: Sämtliche Werke, Band 5, ders., 358-373. München: Hanser.

Schmitt, Marina. 2010. Die Ehebeziehung im mittleren Lebensalter. www.familienhandbuch.de/familieleben/partnerschaft/herausforderung-konflikte/dieehebeziehungimmittlerenerwachsenenalter.php

Schulz, Wolfgang. 1983. Von der Institution der ,Familie‘ zu den Teilbeziehungen zwischen Mann, Frau und Kind. Soziale Welt 4: 401-416.

Schulz, Wolfgang und Florian Pichler. 2005. Lebensqualität in Österreich - ein 20-Jahres Vergleich. In: Österreich zur Jahrhundertwende, hsrg. Wolfgang Schulz, Max Haller, Alfred Grausgruber, 75-113. Wiesbaden: VS Verlag für Sozialwissenschaften.

Shilling, Chris. 2005. The Body in Culture, Technology and Society. London: Sage.

Simmel, Georg. 1997. Sociology of the meal. In. Simmel on Culture, hrsg. David Frisby and Mike Featherstone, 130-136. London: Sage. 
Scramaglia Rosantonietta. 2000. Falling in love and friendship in children and adolescents”. In: Grenzen und Grenzüberschreitungen der Liebe, hrsg. Kornelia Hahn und Günter Burkart, 101-133. Opladen: Leske + Budrich.

Stendhal. 1966. Über die Liebe. München: Winkler.

Sternberg, Robert J. 1988. The Triangle of Love. Intimacy, Passion, Commitment. New York: Basic Books. Sussman, Marvin B. and Suzanne K. Steinmetz. eds. 1987. Handbook of Marriage and the Family. New York/London: Plenum Press.

Veenhoven, Ruut. 1983. The growing impact of marriage. Social Indicators Research 12: 49-63.

Wallerstein, Judith S. und Sandra Blakeslee. 1989. Gewinner und Verlierer. Frauen, Männer und Kinder nach der Scheidung. München: Droemer Knaur.

Weber, Max. 1964. Wirtschaft und Gesellschaft. 2 Bde. Köln/Berlin: Kiepenheuer \& Witsch.

Max Haller, Dr. phil., ist emeritierter Professor für Soziologie der Universität Graz und Mitglied der Österreichischen Akademie der Wissenschaften. Er war Mitbegründer der European Sociological Association und des International Social Survey Programme (ISSP). Forschungsschwerpunkte: Sozialstruktur und soziale Ungleichheit, europäische Integration, international vergleichende Forschung zu gesellschaftlichen Einstellungen und Wertwandel, soziologische Theorie. Er veröffentlichte als Autor und Herausgeber über 30 Bücher und ca. 160 wissenschaftliche Artikel, u.a. in Kölner Zeitschrift für Soziologie, American Sociological Review, Revue Francaise de Sociologie. Als Gastprofessor war er tätig an den Universitäten Innsbruck, Klagenfurt, Wien, Salzburg, University of Californica (Sta. Barbara), Trient (Italien), St. Augustine University of Tanzania. Jüngste Publikation: Ethnic Stratification and Socioeconomic Inequality around the World. The End of Exclusion and Exploitation? Ashgate, Farnham/Surrey (UK) 2015 (unter Mitarbeit von Anja Eder) 